\title{
Rapid odor processing in the honeybee antennal lobe network
}

\author{
Sabine Krofczik ${ }^{1,2}$, Randolf Menzel ${ }^{1,2}$ and Martin P. Nawrot ${ }^{1,2 *}$ \\ Institut für Biologie - Neurobiologie, Freie Universität Berlin, Germany \\ 2 Bernstein Center for Computational Neuroscience, Berlin, Germany
}

\section{Edited by:}

Terrence J. Sejnowski, The Salk

Institute for Biological Studies, USA

Reviewed by:

Collins Assisi, The Salk Institute for Biological Studies, USA

Jing Wang, University of California, USA

\section{${ }^{*}$ Correspondence:}

Martin Nawrot, Neuroinformatics and Theoretical Neuroscience, Institut für Biologie, Freie Universität Berlin, Königin-Luise Str. 28-30, D - 14195

Berlin, Germany.

e-mail: nawrot@neurobiologie. fu-berlin.de
In their natural environment, many insects need to identify and evaluate behaviorally relevant odorants on a rich and dynamic olfactory background. Behavioral studies have demonstrated that bees recognize learned odors within $<200 \mathrm{~ms}$, indicating a rapid processing of olfactory input in the sensory pathway. We studied the role of the honeybee antennal lobe network in constructing a fast and reliable code of odor identity using in vivo intracellular recordings of individual projection neurons (PNs) and local interneurons (LNs). We found a complementary ensemble code where odor identity is encoded in the spatio-temporal pattern of response latencies as well as in the pattern of activated and inactivated PN firing. This coding scheme rapidly reaches a stable representation within 50-150 ms after stimulus onset. Testing an odor mixture versus its individual compounds revealed different representations in the two morphologically distinct types of lateral- and median PNs (I- and m-PNs). Individual m-PNs mixture responses were dominated by the most effective compound (elemental representation) whereas I-PNs showed suppressed responses to the mixture but not to its individual compounds (synthetic representation). The onset of inhibition in the membrane potential of I-PNs coincided with the responses of putative inhibitory interneurons that responded significantly faster than PNs. Taken together, our results suggest that processing within the $L N$ network of the $A L$ is an essential component of constructing the antennal lobe population code.

Keywords: antennal lobe, Apis mellifera, latency code, local interneurons, olfaction, odor mixture, projection neurons, temporal coding

\section{INTRODUCTION}

Honeybees (Apis mellifera) are social insects that use odors as communication signals. These odors are of simple structure, typically composed of a single chemical compound. On the contrary, naturally occurring odorants that become relevant for the bee during foraging are generally complex blends of a large number of volatile compounds (Pichersky and Gershenzon, 2002) with time-varying composition and concentrations. Psychophysical studies in bees (Chandra and Smith, 1998; Deisig et al., 2003), catfish (Valenticic et al., 2000), and humans (Livermore and Laing, 1998) have shown that the neural representation of an odor mixture can be synthetic or elemental whereby the mixture either acquires a novel perceptual quality or it resembles its elemental compounds. In vertebrates, odor mixture interactions were observed at different levels of the olfactory system, in the periphery (Duchamp-Viret et al., 2003), in the olfactory bulb (Davison and Katz, 2007; Giraudet et al., 2002; Lin et al., 2006; Tabor et al., 2004) and in the olfactory cortex (Lei et al., 2006; Zou and Buck, 2006). Studies of mixture interaction in the honeybee antennal lobe using $\mathrm{Ca}^{2+}$-imaging of glomerular activity revealed suppressive interactions in which the response to an odor mixture can be much lower than the response to the single compounds (Deisig et al., 2006; Joerges et al., 1997; Silbering and Galizia, 2007). Such a suppression effect was not observed in the moth Spodoptera littoralis where glomerular Ca responses to mixtures always demonstrated hypoadditive interactions resembling the response to the strongest compounds within the mixture (Carlsson et al., 2007).
Animals have evolved sophisticated mechanisms to distinguish naturally occurring odors, and to extract meaningful information from a rich olfactory environment. Recent behavioral studies could show that a perceptual discrimination of odors occurs rapidly after the onset of an odor stimulation. Mice are able to discriminate dissimilar odors within $<250 \mathrm{~ms}$ (Abraham et al., 2004) and they can distinguish a novel odor from learned odors within only $140 \mathrm{~ms}$ (Wesson et al., 2008). A recent study in honeybees has demonstrated their ability to distinguish between different olfactory stimuli that are as short as $200 \mathrm{~ms}$ (Wright et al., 2009). Consistent with this result, we found that mushroom body (MB) extrinsic neurons in the honeybee reliably signal the presence of a conditioned odor within $<200 \mathrm{~ms}$ after stimulus onset (Pamir et al., 2008; Strube-Bloss et al., 2008). Thus, odor processing in the olfactory pathway must be fast and lead to a reliable code of odor identity at the input level to the central brain structures.

The olfactory system of the honeybee comprises 60,000 olfactory sensory neurons (OSNs; Esslen and Kaissling, 1976), which are located predominantly in pore plate sensillae on the antennae. Each OSN conveys the olfactory information through one of the four antennal tracts (T1-4, Figure 1A) and innervates neurons in one of $\sim 160$ glomeruli (Arnold et al., 1985; Flanagan and Mercer, 1989; Kelber et al., 2006). Glomeruli receiving input from T1 OSN are located in the ventral-rostral hemisphere of the AL whereas glomeruli which receive input from T2-4 glomeruli in the dorsal-caudal hemisphere (Figure 1A; Kirschner et al., 2006). Glomeruli represent synaptic interaction sites between the OSN, 


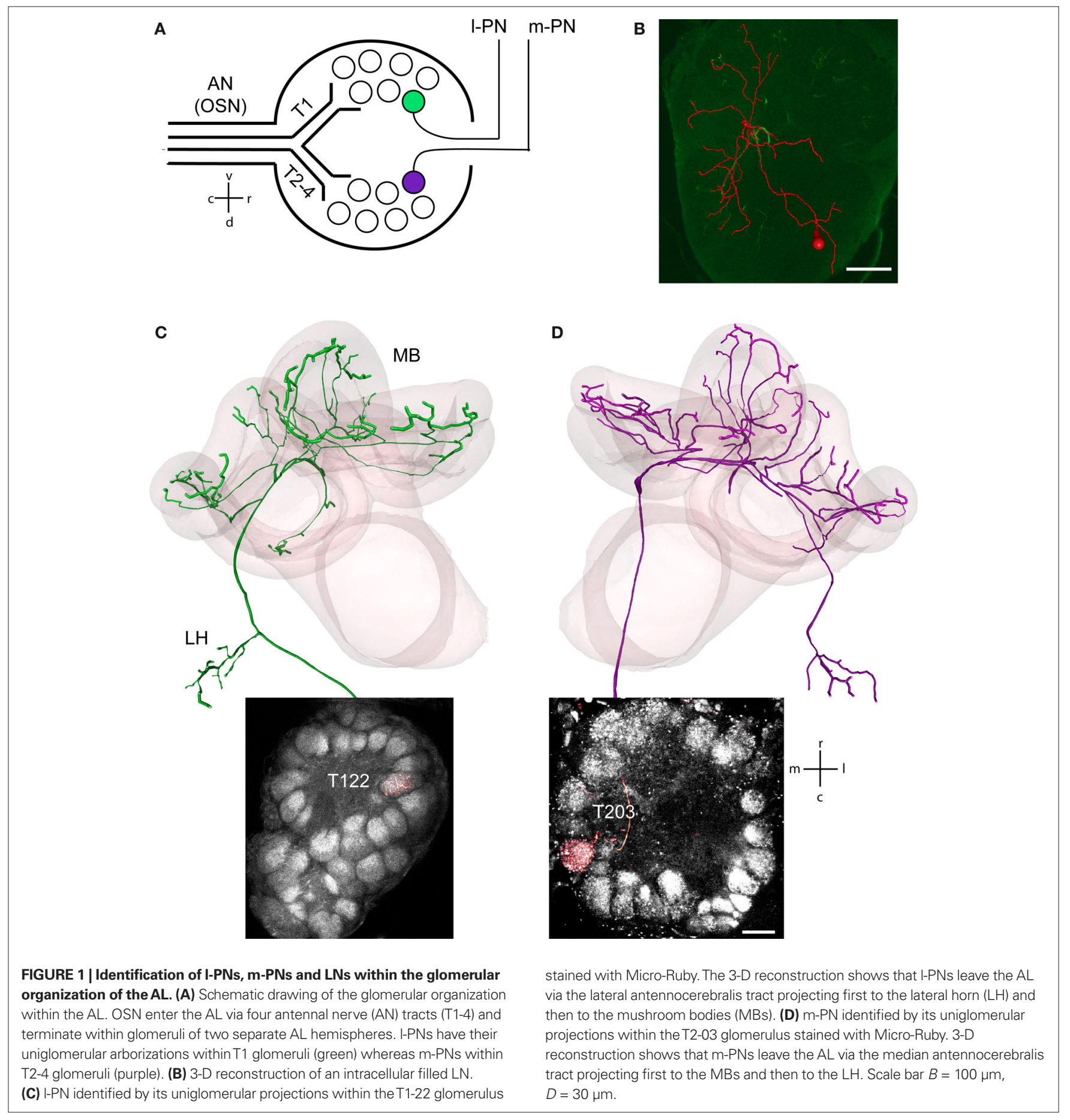

a total of $\sim 4000$ AL-intrinsic local interneurons (LNs, Figure 1B, depicted in red; Fonta et al., 1993) and $\sim 950$ projection neurons (PNs, Figures 1C,D; Kirschner et al., 2006; Rybak and Eichmueller, 1993). Anatomically we distinguish two types of PNs. The lateral PNs (1-PNs) receive input exclusively from T1 glomeruli (Figure $\mathbf{1 A}$, green circle) and send their axons along the lateral antennocerebralis tract to the higher order brain centers, the lateral horn (LH) and the MB. The median PNs (m-PNs) exclusively originate in T2-4 glomeruli (Figure 1A, purple circle) and project along the median antennocerebralis tract, first to the MB and then to the LH (Abel et al., 2001; Bicker et al., 1993; Kirschner et al., 2006; Mobbs, 1982; Müller et al., 2002). Each uniglomerular PN is therefore identified by the respective glomerulus and numbered according to the 3-D Atlas of the honeybee AL (Figures 1C,D; Galizia et al., 1999).

In the present study, we performed intracellular recordings of single l-PNs, m-PNs and LNs and analyzed their spatio-temporal responses to odors and odor mixtures. We specifically addressed the following questions. How fast does an ensemble code evolve 
in a population of PNs? Is there a functional distinction between the two anatomically distinct types of PNs? With regard to mixture encoding we confirmed the hypothesis that l-PNs can show mixture suppression as previously reported in Ca-imaging studies at the glomerular level, and we tested whether this effect does carry over to the m-PNs. Finally, we asked whether local inhibition with in the AL network underlies response suppression in individual PNs.

\section{MATERIALS AND METHODS HONEYBEES}

Worker bees (A. mellifera carnica) were caught at the hive entrance or in an indoor flight room, immobilized by cooling, and mounted in plastic tubes. The bees were fed with sucrose solution and kept in the dark at $20^{\circ} \mathrm{C}$ and high humidity. On the following day, the head was fixed with wax and opened between the median ocellus and the base of the antennae. Glands and tracheal sheaths were removed. A second hole was cut to expose the esophagus and to stretch it aiming to reduce movement of the brain. The brain was kept wet at all times by dribbling drops of bee physiological saline solution onto (in $\mathrm{mmol}^{-1}$ : $130 \mathrm{NaCl}, 6 \mathrm{KCl}, 2 \mathrm{MgCl}_{2}, 10 \mathrm{HEPES}$, 17 glucose, 6 fructose, 160 sucrose, $\mathrm{pH}$ 6.7).

\section{ELECTROPHYSIOLOGY}

Glass electrodes were pulled with a horizontal laser puller (P-2000 Sutter Instruments, Novato, CA, USA) and their tips were filled with $4 \%$ tetramethylrhodamine-biotin dextran (Micro-Ruby; Invitrogen, Germany) in $0.2 \mathrm{M}$ potassium acetate. The electrodes (resistance 120-200 M $\Omega$ ) were positioned at the top of the AL by using a micromanipulator (Leitz) and lowered posteriorly into the neuropil until a neuron could be penetrated. A reference electrode, a chlorized silver wire, was inserted into the eye. The recordings were done in bridge mode using an intracellular BRAMP 1 amplifier (NPI Electronics, Tamm, Germany). Data were, digitized using a 1401 interface and stored on a PC using spike2.5 software (Cambridge Electronic Design, UK).

\section{NEURON IDENTIFICATION}

Single l- and m-PNs were intracellular stained by injecting MicroRuby iontophoretically using depolarizing current pulses with a duration of $0.2 \mathrm{~s}$ applied at $1-2 \mathrm{~Hz}$. Successful staining allowed the identification of the PN type ( 1 or $\mathrm{m}$ ) by location of their uniglomerular projections (Figures 1C,D) (1-PNs receive input from T1 glomeruli whereas m-PNs from T2-T4 glomeruli, see Figure 1A). Intracellular recording and filling (approximate recording time $>10 \mathrm{~min}$, approximate staining time $\sim 20 \mathrm{~min}$ ) was performed intra-axonally. Due to the small axon diameter $(-3-5 \mu \mathrm{m})$, recording conditions were extremely difficult, strongly limiting the overall experiment time. We kept stimulus protocols short to ensure sufficient time for the iontophoresis. In cases of highly stable recording conditions, we increased the stimulus protocol to either comprise a larger stimulus set or to increase number of repetitions for a subset of the stimuli. Successful staining could be achieved in about $50 \%$ of all neurons. Since the antennal lobe of the honeybee is well described (Abel et al., 2001; Galizia et al., 1999; Kirschner et al., 2006) identification of l- and m-PNs was alternatively performed by the spatial position of the electrode with respect to the hemispherical segregation of T1 and T2-4 glomeruli
( T1 = ventral-rostral, T2-4 = dorsal-caudal). LNs could easily be identified either by their specific intracellular action potential waveform and their firing pattern which clearly differ from those of the PNs (Galizia and Kimmerle, 2004). Previous imaging studies of the AL could only resolve signals from the T1 glomeruli but not form T2-4. We therefore preferentially targeted $\mathrm{m}-\mathrm{PNs}$ that innervate T2-4. In total we identified and analyzed PNs: $N=7$ (1-type), $N=23$ (m-type); and LNs: $N=7$.

\section{ODOR STIMULATION}

The animal's antennae were exposed to a constant active charcoalfiltered stream of room air (airflow rate $10 \mathrm{ml} / \mathrm{s}$ ) guided through a glass tube with an inner diameter of $14 \mathrm{~cm}$ placed $1 \mathrm{~cm}$ from the antenna. For each odor, $4 \mu \mathrm{l}$ were placed on a filter paper in a glass syringe, which was introduced into the continuous air stream. The control stimulus was a glass syringe plus filter paper with mineral oil (Sigma-Aldrich, Germany) delivering a constant airflow rate of $10 \mathrm{ml} / \mathrm{s}$. To avoid mechanical stimulation a computer-controlled valve switched between control stimulus and odor-loaden syringe such that the antenna was always exposed to an air stream with a flow rate of $20 \mathrm{ml} / \mathrm{s}$. The stimulus timing was computer-controlled and each odor stimulation lasted for $2 \mathrm{~s}$. The delay from the onset trigger for valve opening to the arrival of the odorant at the antenna was estimated as $\sim 200-220 \mathrm{~ms}$. For response latency analyses and display purposes we therefore subtracted a fix delay of $200 \mathrm{~ms}$ from the measured latencies. All odors were diluted 1:10 in mineral oil and checked for purity and mixture composition by gas chromatography (Trace GC Ultra, Thermo, Electron Corporation, Waltham, MA, USA). The animals' antennae were stimulated with a tertiary mixture and its elemental compounds as well as other single compound odors and complex odor mixtures (Sigma-Aldrich, Germany). The tertiary mixture was composed of the blossom compounds nonanol and hexanol and 2-heptanon, which is known to be a compound of the alarm pheromone in bees (Balderrama et al., 2002). Additionally natural floral blends as well as single compounds exhibiting different functional groups were tested. In some animals multiple-trial presentations of the same odor were used to test response reliability, whereas in other animals one-trial presentation (in some cases with few repetitions) of different odors (between 2 and 30) were performed in a randomized fashion. We analyzed only stable recordings that lasted for at least $10 \mathrm{~min}$.

\section{DATA ANALYSIS}

Matlab and the Matlab Statistics Toolbox (The MathWorks, Inc.) were used for the analysis of the intracellular voltage traces and spike trains. We implemented all custom-written algorithms used for analysis in the present manuscript in the FIND open source Matlab toolbox for neural data analysis (Meier et al., 2008; http:// find.bccn.uni-freiburg.de/).

\section{Firing rate analysis}

Intracellular spikes times were detected with a threshold criterion. Firing rates were measured by convoluting single trial spike trains with a kernel of asymmetric shape $k(t)=t \times \exp (-t / \tau)$ and time resolution $\tau$, defined on a finite support (Nawrot et al., 1999; Parzen, 1962). We aligned the resulting rate function at time $w$ that indicates the center of mass of the kernel ( $w=42 \mathrm{~ms}$ for $\tau=25 \mathrm{~ms}$ ). For 
color-coded representation of excitatory and inhibitory responses, we subtracted from the response the baseline rate measured during $1 \mathrm{~s}$ preceding the stimulus. We then represent the rate response of a neuronal ensemble with $n$ neurons to odor stimulus $a$ at any given point in time as the $n$-dimensional rate vector $v^{a}$. We then define the distance between two rate vectors $v^{a}, v^{b}$ as $d_{a b}=\Sigma\left|v_{i}^{a}-v_{i}^{b}\right|$.

\section{Response latency analysis}

To estimate absolute and relative trial-by-trial latencies we used a method that we described in detail elsewhere (Meier et al., 2008). A demo script for this method together with a demo data set is available online at the official website of the FIND toolbox (http://find. bccn.uni-freiburg.de/?n=Docu.Tutorial). In a first step, we estimated from a given single trial spike train the time derivative of the signal by convolution with an asymmetric Savitzky-Golay filter (Savitzky and Golay, 1964) (polynomial order 2, width $300 \mathrm{ms,}$ Welch windowed). In a second step, we estimated the N-1 relative latencies of the individual spike trains. For this we used an algorithm that allows to optimally align all $N$ single trial derivatives (taking into account the first $700 \mathrm{~ms}$ ) such that their pair-wise cross-correlation is maximized (Nawrot et al., 2003). The resulting optimal N-1 time-shifts correspond to the relative latencies and we measured their standard deviation $\sigma$ to quantify the cross-trial latency variability. In a third step, we aligned in time all individual single trials according to the optimal time shifts (with a zero mean shift to preserve the center of mass) and then merged all single trial spike trains into 1 .

For each odor, we thus obtained one trial-aligned and merged spike train. To obtain the relative latencies across different odors we repeated the same procedure but now using the merged spike trains. We again used the standard deviation to quantify latency variability across odors. To test for the significant difference of two distributions of latency differences we used the Kolmogorov-Smirnov test. To obtain the absolute response latency for each neuron as averaged across all odors we optimally aligned the odor-specific spike trains as described above and merged all spike trains. We then computed the time derivative of the firing activity as explained above and measured the peak time of the maximum. We defined this point in time at which the rise of the response was steepest as the absolute single neuron latency. Absolute latency estimates have a small but fix bias toward slightly higher values as we subtracted the lower limit of the estimated delay for odor delivery $(200 \mathrm{~ms})$, and because of the asymmetry of the Savitzky-Golay filter kernel.

Before estimating the latency of intracellular inhibition the membrane potential of PNs was low-pass filtered with a cut-off frequency of $100 \mathrm{~Hz}$ using an exponential impulse response kernel (decay time constant $1.6 \mathrm{~ms}$ ). Response onset was then determined as the time of crossing a threshold defined as the average baseline response (during $5 \mathrm{~s}$ preceding stimulus onset) minus either one or two times the standard deviation during baseline period (excluding APs).

\section{Specificity index}

We defined the specificity index $s$ of a neuron as the number of odors that evoked either an excitatory $\left(s_{\text {ex }}\right)$ or inhibitory $\left(s_{\text {in }}\right)$ response (within the first second after stimulus onset) divided by the total number of tested odors. For each type of PNs we computed the specificity index separately for single compound and odor mixture presentations.

\section{Mixture index}

To quantify the response difference between mixture and most effective compound we calculated the mixture index $\kappa=(y-x) /$ $(x+y)$ where $x$ denotes the absolute number of response spikes for the individual odor compound that yielded the strongest response and $y$ denotes the absolute number of spikes in the response to the mixture, counted in a fix interval from 200 to $700 \mathrm{~ms}$ after stimulus onset at $t=0$. $\kappa$ Will be close to 0 in the case of a hypoadditive mixture response, and $\kappa$ assumes values close to $-1(+1)$ in the case of a suppressive (additive) mixture effect. The distribution of $\kappa$ values may be strongly skewed, in particular for values close to $-1 / 1$. Therefore, before averaging values of $\kappa$ we applied Fisher's $z$-transform. We tested for a deviation from the null hypothesis of two samples of $\kappa$ having the same median using the Wilcoxon rank sum test.

\section{RESULTS}

\section{I- AND m-PN ODOR RESPONSE PROFILES}

We performed intracellular recordings of single PNs and tested their dynamic responses elicited by single compound odors. 1- and $\mathrm{m}$-PNs were identified either by their uniglomerular arborizations, (Figures 1C,D) or by the location of the particular recording site (see Section "Materials and Methods"). First, we characterized the response kinetics and response reliability of PNs. Both types of PNs typically exhibited a phasic-tonic spike response pattern that outlasted the stimulus presentation. Figures $2 \mathrm{~A}-\mathrm{D}$ shows the response characteristics for a l-PN receiving input from the T1-22 glomerulus (Figures 2A,B) and a $\mathrm{m}-\mathrm{PN}$ receiving input from the T3-45 glomerulus (Figures 2C,D). The responses were highly reliable across trials in all neurons tested. If a response was observed during one stimulus presentation, then it could reliably be detected in all of the trials. This is demonstrated in the spike raster diagrams of Figures 2B,D. Based on this result we reduced the odor stimulus protocol to single or few trials of one and the same odor to test a larger set of odors within the limited experimental time. On average, the dynamic response profile of 1 - and $\mathrm{m}$-PNs was very similar (Figure 2E). The spontaneous firing rate was typically low with a mean of 6.8 spikes/s (median 3.4/s) and the average maximum responses peaked around 70 spikes/s. Several neurons showed no spontaneous spiking activity. Average response latencies of individual neurons were not significantly different $(P=0.7$, Wilcoxon rank sum test) in l-PNs $(148.3 \pm 33 \mathrm{~ms})$ and $\mathrm{m}-\mathrm{PNs}(123.5 \pm 14.6 \mathrm{~ms})$ (Figure 2F).

\section{ODOR-SPECIFIC RESPONSE LATENCIES OF I- AND m-PNs}

From behavioral experiments, it becomes evident that a code of odor identity must be rapidly established at the level of the AL output (see Section "Introduction"). We here consider one possible fast coding strategy, namely the encoding of odor identity in an odor-specific pattern of response latencies across an activated $\mathrm{PN}$ ensemble. To this end, we compared the differences in response latency as measured across different odors with the differences in latency across trials of repeated stimulus presentation. The experimental limitations of recording time made it necessary 
A

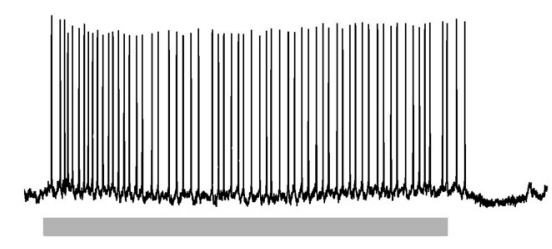

B
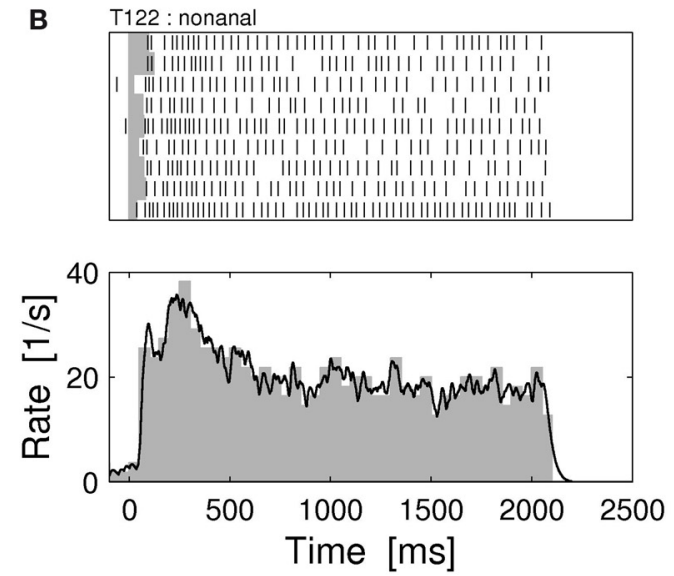

E

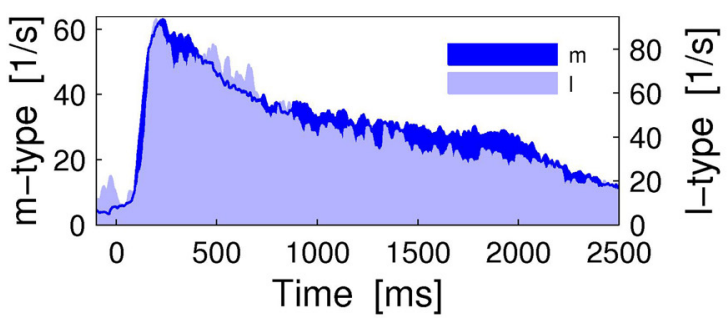

C

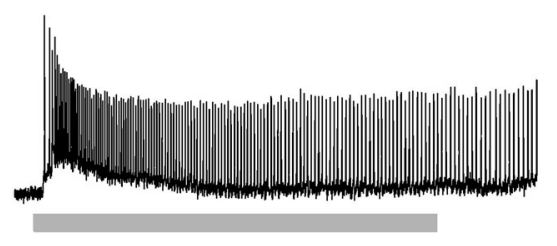

D T345: heptanone
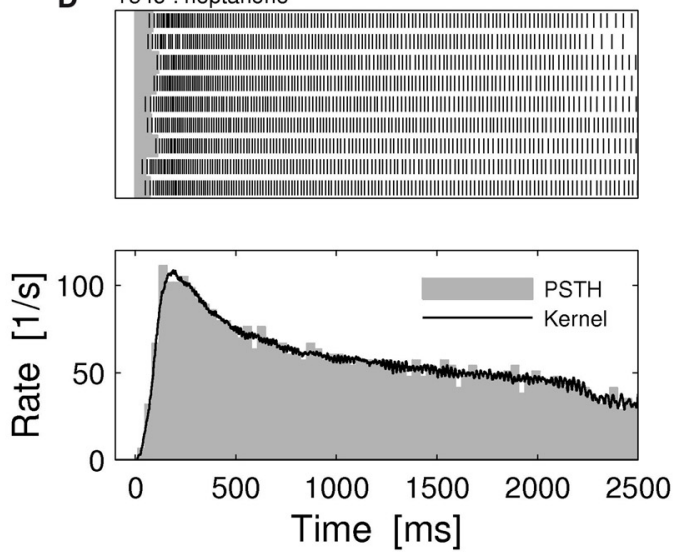

$\mathbf{F}$

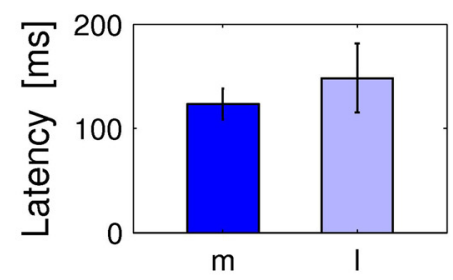

FIGURE 2 | Odor response dynamics of projection neurons. (A) Intracellular membrane potential of one I-PN in response to the first presentation of the odor nonanal for $2 \mathrm{~s}$ (gray bar). Intracellular staining revealed that this neuron innervated the glomerulus T1-22. (B) The neuron responded reliably in all repeated presentations of the same stimulus (top). The individual single-trial latencies of response onset were estimated as indicated by the horizontal gray bars. The trial-averaged rate profile (bottom) shows a phasic-tonic response dynamics. (C) Intracellular response to 2-heptanon during the first trial and
(D) repeated reliable responses of an identified m-PN that innervated the glomerulus T3-45. The rate profile indicates the initial phasic response followed by a tonic response that lasted throughout the static stimulus. (E) The average response rate profiles are of similar shape for $\mathrm{I}(N=7)$ and $\mathrm{m}-\mathrm{PNs}(N=23)$. (F) The response latencies of I-PNs are not significantly different from those of $\mathrm{m}-\mathrm{PNs}(P=0.7$; Wilcoxon rank sum test). Average response latencies: $148.3 \pm 33 \mathrm{~ms}(\mathrm{I}-\mathrm{PN})$ and $123.5 \pm 14.6 \mathrm{~ms}$ (m-PN). PSTH bin width: $20 \mathrm{~ms}$; kernel resolution: $\tau=14 \mathrm{~ms}$. that we typically tested a diverse set of odors with very few trials or only very few odors during many trials. For a statistical evaluation, we therefore pooled all relative across-trial latencies (see Section "Materials and Methods") separately for 1- and m-PNs (Figures 3A,B). The trial-to-trial variability of response onset was quantified by its standard deviation $\sigma$ which was similar for both, l-PNs (Figure 3A) and m-PNs (Figure 3B) with $\sigma_{l}^{\text {trial }}=22.5 \mathrm{~ms}$ and $\sigma_{m}{ }^{\text {trial }}=28.3 \mathrm{~ms}$, respectively. Next, we quantified the relative latencies across odors for each neuron and then pooled these again separately for the two types of PNs. The standard deviation of latencies across odors was considerably larger with $\sigma_{1}^{\text {odor }}=41.6 \mathrm{~ms}$ (Figure 3A) and $\sigma_{m}{ }^{\text {odor }}=49.2 \mathrm{~ms}$ (Figure 3B). These latency differences across odors are significantly larger than across trials in both, 1- and m-PNs (see Section "Materials and Methods"). Thus, on a statistical basis, response onset is odor-specific in both types of PNs. For the population of PNs this result immediately implies that there exists an odor-specific spatial latency pattern in the activated ensemble of uniglomerular PNs (cf. Figure 4A) establishing a fast code of odor identity.

\section{I- AND m-PN RESPONSES IN SPACE AND TIME}

Results from $\mathrm{Ca}^{2+}$ imaging studies in the honeybee AL have shown odor-specific activation pattern of T1-glomeruli which are innervated by 1-PNs. This suggests that odor identity is encoded by the activation of an odor-specific ensemble of PNs (Galizia and Menzel, 2000; Joerges et al., 1997; Sachse et al., 1999). To test this hypothesis on the level of individuall- and m-PNs we performed two analyses. First, we concentrated on individual PNs and quantified their odor selectivity on the basis of mean responses during the first $800 \mathrm{~ms}$ after stimulus onset. For l- and m-PNs separately, we classified each response as inhibitory, excitatory or no response and defined the specificity index $s$, which represents the relative number of odors that elicited an excitatory $\left(s_{\mathrm{ex}}\right)$ or inhibitory response $\left(s_{\mathrm{in}}\right)$ (see Section "Materials and Methods"). Our results show that on 

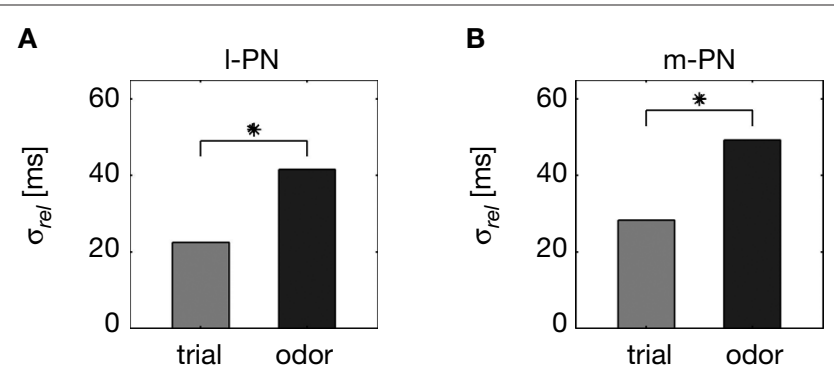

FIGURE 3 | Odor-specific response latencies in projection neurons. Standard deviation $\sigma$ of across-trial latencies (gray) and across-odor latencies (black) in I-PNs (A) ( $N=62 / 29)$ and m-PNs (B) $(N=96 / 67)$. The differences of latencies across odors are significantly larger than the differences across trials ( $P<0.01$; Kolmogorov-Smirnov) for both types of $\mathrm{PNs}$ as indicated by the star in $(\mathbf{A}, \mathbf{B})$. The differences in latencies across trials were not significantly different for the two types of PNs $(P>0.05)$. See Section "Materials and Methods" for details on latency estimation.

Table 1 | Specificity index for single compound odors and odor mixtures for different neuron types. Relative number of single compound odors (left) and odor mixtures (right), which elicited excitatory $\left(s_{\text {ex }}\right)$ or inhibitory spike responses $\left(s_{\text {in }}\right)$, averaged across all neurons. I-PNs $(N=7)$ showed fewer excitatory responses than $\mathrm{m}-\mathrm{PNs}(N=23)$, but in addition exhibited inhibitory response to single compound odors and odor mixtures. Such inhibition was virtually absent in $\mathrm{m}-\mathrm{PNs}$. LNs $(N=7)$ are rather unspecific and responded to $3 / 4^{\text {th }}$ of the tested mixtures. On average, we tested 4.3 single compounds and 3.7 complex odors per neuron.

\begin{tabular}{|c|c|c|c|c|}
\hline & \multicolumn{2}{|c|}{ Single compound } & \multicolumn{2}{|c|}{ Mixture } \\
\hline & $s_{\text {ex }}$ & $S_{\text {in }}$ & $S_{\text {ex }}$ & $S_{\text {in }}$ \\
\hline I-PN & 0.56 & 0.13 & 0.45 & 0.12 \\
\hline m-PN & 0.65 & 0.07 & 0.64 & 0.00 \\
\hline LN & 0.60 & 0.00 & 0.75 & 0.00 \\
\hline
\end{tabular}

average 1-PNs show an excitatory response to about half of the odors tested while m-PNs are less odor-specific with excitatory responses to about $2 / 3^{\text {rd }}$ of the tested odors (cf. Table 1 ; for examples see Figure 1 in Supplementary Material). Inhibitory responses (i.e., a suppressed spike rate) were observed in 1-PNs for either $12 \%$ or $13 \%$ of the odors but they were very rarely observed in $\mathrm{m}$-PNs and only for single compound odors but never in the case of an odor mixture. The odor-specific mean response classification of individual neurons necessarily translates into an odor-specific binary response pattern across the $\mathrm{PN}$ population.

Next, we examined the spatio-temporal activation in a pseudo population of non-simultaneously recorded 1 - and $\mathrm{m}$-PNs and their responses to three single compound odors, the ketone 2-heptanon (2,7on) and the alcohols hexanol (6ol) and nonanol (9ol) (Figure 4A). The color-coded response rate profiles in Figure 4A indeed indicate that there exists a unique spatio-temporal pattern associated with each odor. A mean response classification (excitatory, inhibitory, no response) resulted in an odor-specific fingerprint (matrix in Figure 4A). Since responses were highly reliable across repeated stimulations, this odor-specific spatial activation pattern will be identical in each trial. To investigate how the separations of odor-specific ensemble firing patterns evolve over time we computed the vector distance $d$ in time-resolved fashion. In each point in time $d_{a b}$ measures the distance between the vector of neuronal firing rates for two odors $a$ and $b$. In each panel of Figure 4B we show the average distance $d^{\prime}=\left(d_{a b}+d_{a c}\right) / 2$ between the respective odor $a$ and the two other compounds. The units of $d$ represent the difference in firing rate per neuron, i.e., the number of spikes per second per neuron (see Section "Materials and Methods"). The resulting profiles in Figure 4B display a highly dynamic behavior. The vector distance increases rapidly after stimulus onset and peaks at about 150 250 ms. The highest divergence of odor response patterns naturally occurs only during the initial phasic responses. After about 300-500 ms the patterns again converge to reach an almost constant distance of $\sim 20$ spikes/s associated with the tonic response that outlasted the stimulus.

The spatial identification of 1 - and $\mathrm{m}$-PNs by their uniglomerular arborizations further allowed us to establish a schematic map of the glomerular activation patterns within the AL. In Figure $4 \mathrm{C}$ the spatio-temporal firing rate of identified $\mathrm{PNs}$ during the stimulation with 2-heptanon (2,7on) is shown as a glomerular activation pattern in eight successive time windows. Each time window represents in a color code the odor response for each individual PN at the location of the corresponding innervated glomerulus, resulting in a spatio-temporal activation pattern across all identified glomeruli. This interpretation assumes that the average $\mathrm{PN}$ activity of several uniglomerular neurons is highly similar to the response profile of the individual recorded neuron. Conversely, such a mapping can make a direct prediction for the glomerular response pattern in Ca-imaging studies that measure the mean activity of identified glomeruli.

\section{DIFFERENTIAL ODOR MIXTURE INTERACTIONS IN I- AND m-PNs}

Imaging studies in the honeybee AL revealed that T1-glomeruli often exhibit suppressed responses to a mixture but not to the elemental mixture components when presented alone (see Section "Introduction"). To test the prediction that this suppression effect is also evident at the level of single l-PNs, and to test whether it is also observable in individual $\mathrm{m}$-PNs, which are not accessible by Ca-imaging in the $\mathrm{AL}$, we compared individual l- and $\mathrm{m}-\mathrm{PN}$ responses to an odor mixture and to its three elemental components when presented alone. Figures 5 A,B show the dynamic odor response profiles of one 1- and one $\mathrm{m}-\mathrm{PN}$ elicited by the tertiary mixture (black) and its compounds, the ketone 2-heptanon (green), and the alcohols hexanol (orange) and nonanol (blue). The l-PN showed excitatory responses to all three elemental compounds but was suppressed by their tertiary mixture (Figure 5A). Note that the suppression is rapid such that not a single response action potential is elicited. The $\mathrm{m}-\mathrm{PN}$, however, showed excitatory responses to the odors hexanol, 2-heptanon and to the mixture, where the response to the mixtures resembled the stronger response to 2-heptanon (Figure 5B).

The color-coded response profiles in Figure 5C show the odor mixture responses across all PNs tested. The 1-PNs were mostly inhibited or showed no response. In comparison, the majority of m-PNs showed a pronounced excitatory response. 


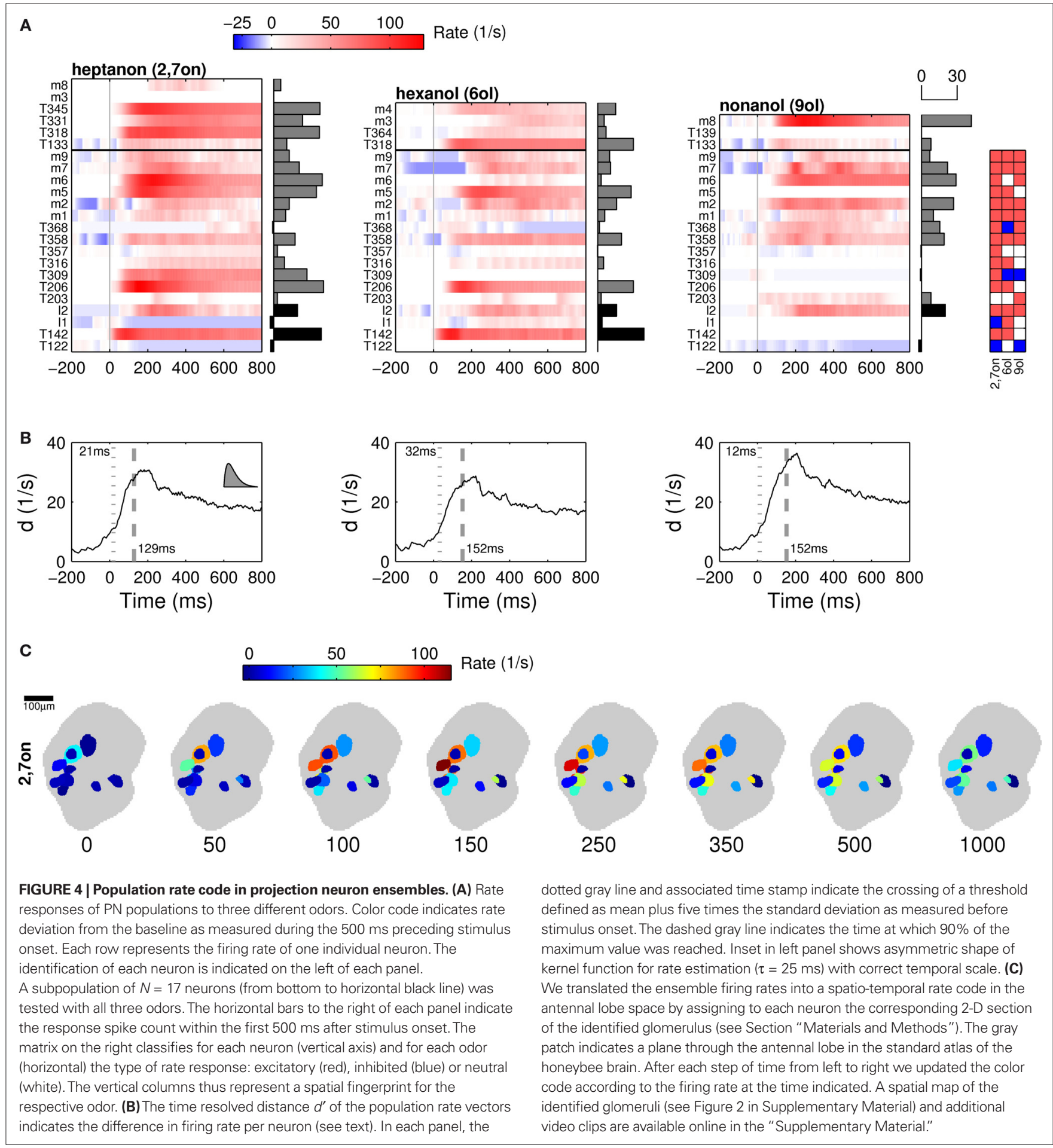

In Figure 5D, we show the time resolved vector distance $d$. In each point in time, it measures the difference of the neuronal ensemble response to the mixture and its response to each of the individual compounds (cf. Figure 4) as indicated by line color. The spatial response pattern for the mixture rapidly and strongly diverged from the response patterns of hexanol (orange) and nonanol (blue), and to a lesser degree from the response pattern of 2-heptanon (green).

We classified odor mixture responses according to three categories of mixture interactions: higher (synergy), equal (hypoadditivity) or lower (suppression) than the response to the most effective compound, following the conventions in (Duchamp-Viret et al., 2003). For each l- and m-PN we determined the most effective compound, i.e., with the strongest response, and compared it with the response to the odor mixture. The bar diagram in Figure 5E shows the response magnitude elicited by 


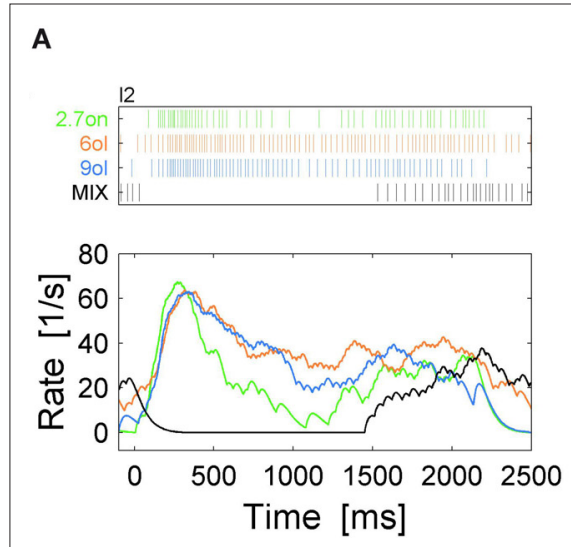

B
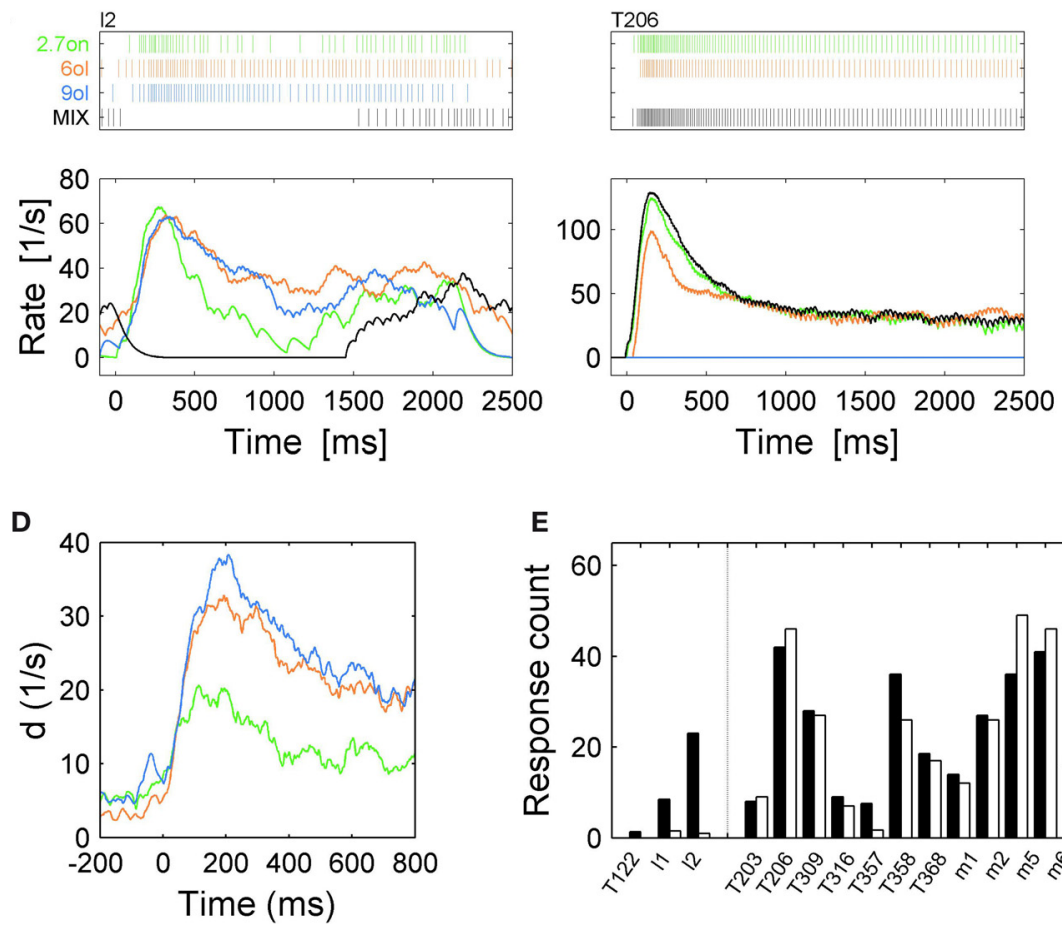

E

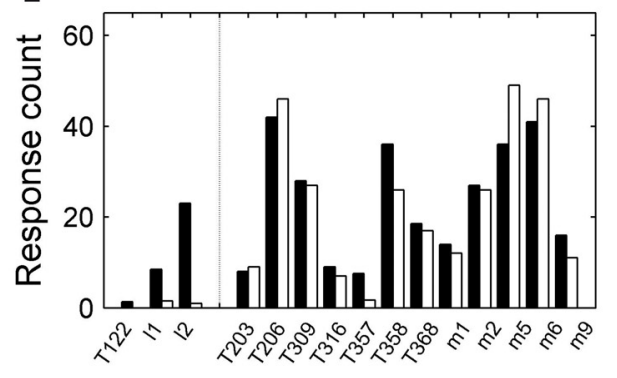

C

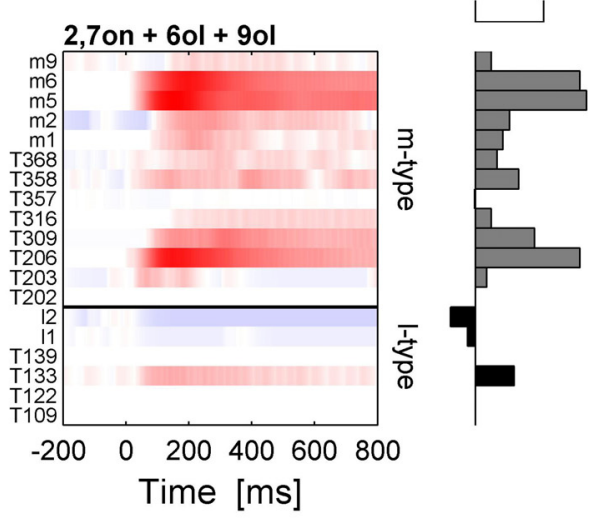

$\mathbf{F}$

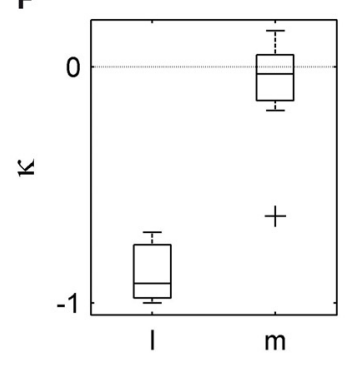

FIGURE 5 | Different mixture effects in l- and $\mathbf{m}$-PNs. (A) Single trial spike trains (top) and rate responses (bottom) of an I-PN to three odor compounds (heptanon $=$ green, hexanol $=$ orange, nonanol $=$ blue) are excitatory while the response to the mixture (black) of all three odors is inhibited. (B) An example of an $\mathrm{m}-\mathrm{PN}$ that shows an excitatory response to the same mixture (black) which closely resembles the most salient response to the elemental mixture compound heptanon. (C) Color-coded rate responses of $6 \mathrm{I}-\mathrm{PNs}$ and $13 \mathrm{~m}-\mathrm{PNs}$ (separated by the blue horizontal line) in response to the same tertiary mixture. The I-PNs show mostly no or inhibited responses while the m-PNs show clear excitatory responses as indicated by the horizontal count bars. (D) Dynamics of the distance $d$ between the ensemble response to the mixture and the ensemble response to each of the mixture elements for 15 neurons that were tested for all four odors. (E) Comparison of the absolute count measured during an initial response interval of $500 \mathrm{~ms}$ in response to the strongest compound (black) and the response count for the mixture (white) for all neurons that were tested in all conditions. The I-PNs indicate inhibited responses to the mixture, while the m-PNs indicate strong excitatory responses to the mixture. (F) The index $\kappa$ measures the relative difference of mixture response and most salient response to an individual compound for all $\mathrm{I}$ - and $\mathrm{m}$-PNs that were tested in all conditions. the odor mixture (white bars) and its strongest compound (black bars) indicating clear suppressive odor mixture interactions for all three l-PNs that we had tested for all compounds. In contrast, hypoadditive odor mixture interaction was observed in all m-PNs. For a quantitative analysis we determined the mixture interaction index $\kappa$ as the relative difference of response magnitude between the mixture and the strongest compound (see Section "Materials and Methods"). The $\kappa$ index reveals either synergistic (1), hypoadditive $(0)$ or suppressive $(-1)$ odor mixture interaction in each l- and $\mathrm{m}-\mathrm{PN}$ tested. The 1-PNs showed a clear suppressive interaction $(\kappa=-0.98)$ in line with the prediction from imaging studies. Surprisingly, the $\mathrm{m}-\mathrm{PNs}$ exhibited a clear hypoadditive interaction with average $\kappa=-0.09$ (Figure 5F). This difference between 1 - and $\mathrm{m}-\mathrm{PN}$ mixture interactions was significant $(P<0.01$, Wilcoxon rank sum test). Synergistic odor mixture interactions were found in neither of the tested PNs. In Table 1 we listed the specificity index separately for single chemical compounds and mixtures that combined at least three such elementary compounds. It shows that only l-PNs exhibited inhibited responses to mixtures in $12 \%$ of all cases. Thus, our results for the l-PNs meet the prediction of previous imaging studies. The fact that we did not observe any suppressed response in all our $\mathrm{m}-\mathrm{PN}$ recordings is a surprising and unexpected result, marking a functional difference of the two types of PNs.

\section{FAST INHIBITION IN I-PNS COINCIDES WITH LN RESPONSES}

What could be the cause for the observed response suppression in 1-PNs following odor mixture stimulation? Does suppression become effective at the level of OSNs or could local inhibition within the AL network explain the effect? To answer this question we first tested the intracellular membrane potential of PNs for signs of inhibition. The experimental setting for intracellular recordings from PNs makes it necessary to record from their individual axons. Thus, we recorded the membrane potential distal from the integrating segments and individual synaptic inputs could not be resolved. APs, however, were always detectable and in some cases we could observe clear hyperpolarizing deviations of the membrane potential that can be attributed to inhibitory inputs. In Figure 6A we show six example traces from 1-PNs with marked inhibition following stimulus onset. We can now see that the l-PN that innervates the T1-22 glomerulus is actually inhibited in response to the previously examined tertiary mixture. This was 


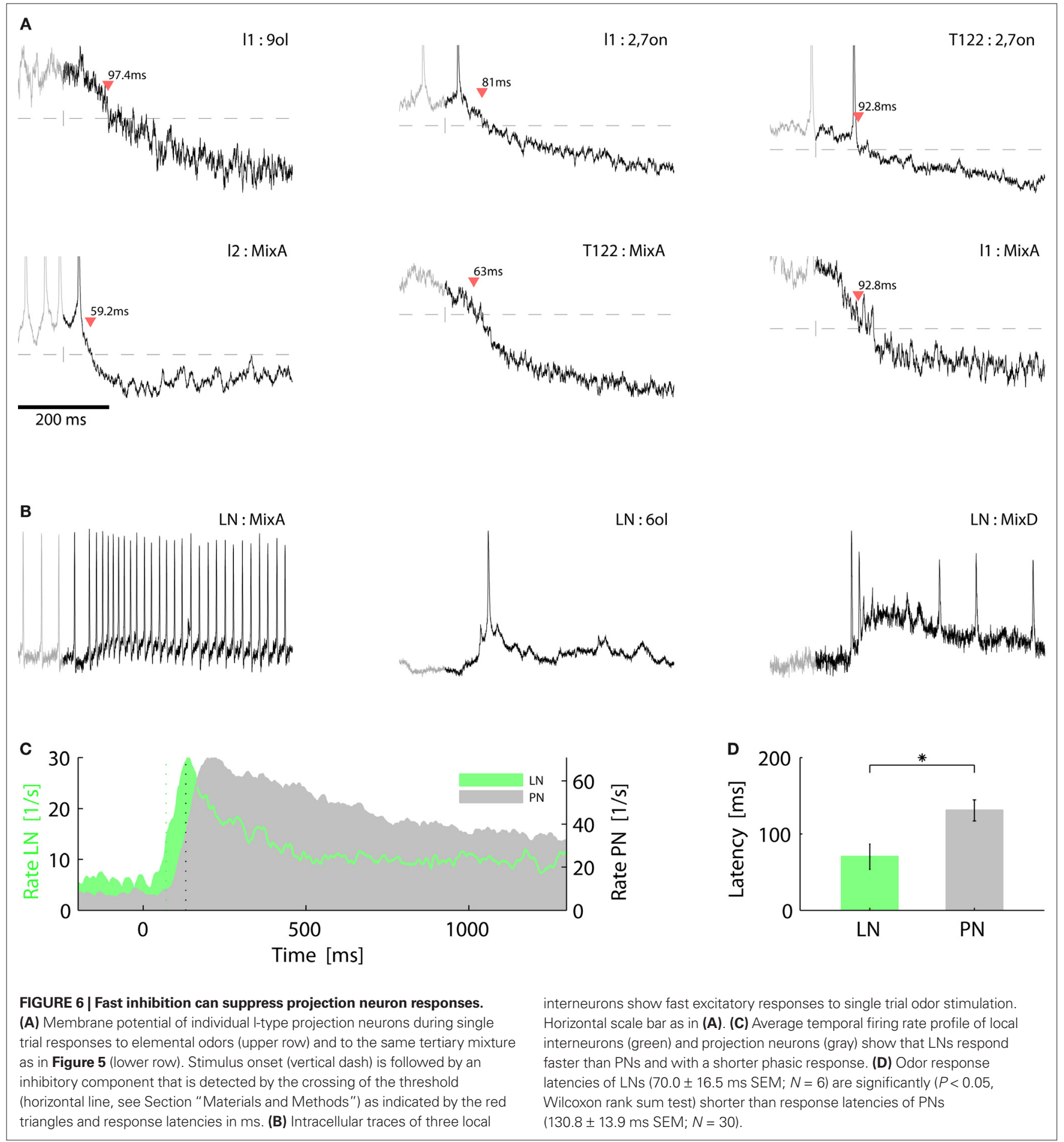

not visible in the rate response in Figure $5 \mathbf{C}$ because of the low spontaneous activity level. We estimated the onset of the inhibitory compound by thresholding the membrane potential with respect to the mean and standard deviation prior to stimulus onset (see Section "Materials and Methods"). Surprisingly, we found that the response latency of the observed inhibition is on the order of only $\sim 60-90 \mathrm{~ms}$ and thus considerably shorter than the observed excitatory spike responses in PNs (Figure 2). This explains why during mixture response suppression we did not observe a single response spike even in the initial response phase.

Could this fast inhibition of PNs be mediated by LNs? To answer this question we analyzed the onset latencies of excitatory spike responses in LNs. Figure 6C shows the average dynamic rate profile of LNs (green) and PNs (gray). Indeed, the average latencies (vertical dotted lines) of LNs are significantly shorter than those of PNs with an average delay of $\Delta t \approx 60 \mathrm{~ms}$ (Figure 6D). The fast 
excitatory response of LNs to odor onset is also evident in the individual single trial traces shown in Figure 6B. We may conclude that inhibitory interneurons in the AL can exhibit fast odor responses and are thus capable of rapidly and effectively suppressing $\mathrm{PN}$ responses.

\section{DISCUSSION}

We investigated the nature and dynamics of the encoding of single compound odors and odor mixtures in two morphological distinct types of PNs of the honeybee using in vivo intracellular recordings. Our results show that each odor activated a subset of PNs with each $\mathrm{PN}$ responding rather non-specifically to a number of odors. The spatial representation of odor identity in the PN ensemble evolves rapidly and peaks at about $150 \mathrm{~ms}$ after stimulus onset. PN activation involves a distinct odor-specific response latency, which implies an odor-specific pattern of relative latencies across the activated $\mathrm{PN}$ ensemble that is conveyed to higher order olfactory neuropils, the $\mathrm{MB}$ and the $\mathrm{LH}$. Odor mixture representation depends on the type of PN, signifying a functional specialization of the two anatomically distinct neuron types. Our results indicate that 1-PNs encode odor mixtures synthetically through a suppressed response to the mixture - an observation that was previously made on the level of average glomerular activity using Ca-imaging techniques - whereas m-PNs were never suppressed but encoded odor mixtures elementally, representing the strongest compound within the mixture (hypoadditive mixture interaction). Moreover, our results showed that synthetic odor mixture representation is associated with a prolonged hyperpolarization of the membrane potential, the onset of which in turn matched the stimulus-response latencies of LNs, which are significantly and considerably shorter than the ones observed in PNs. Therefore, we postulate that suppressive odor mixture interactions as observed in 1-PNs arise from fast lateral inhibition mediated by the LN network.

\section{COMPARISON WITH IMAGING STUDIES}

Part our results show that excitatory single neuron responses to individual odors organize into spatial patterns that involve about half of the PN population. Mixture suppression and inhibitory responses to complex odorants were confined to l-PNs. This part of our results is consistent with earlier imaging studies that could demonstrate the spatial representation of odors across T1 (1-PN) glomeruli in the honeybee AL (Deisig et al., 2006; Galizia et al., 1999; Joerges et al., 1997; Sachse et al., 1999) and suppressive odor mixture interaction in T1 glomeruli (Deisig et al., 2006; Guerrieri et al., 2005; Joerges et al., 1997). However, the Ca-imaging methods used to monitor AL activity imposed several technical limitations. First, Ca-imaging yields only a limited temporal resolution that makes it difficult to assess the detailed dynamics with which a reliable representation emerges in the glomerular space. Second, during bath application of the Ca dye one generally cannot distinguish the types of neurons that were stained, PNs, LNs (excitatory or inhibitory), or glia (Heil et al., 2007). Retrograde staining methods are likely to stain several PNs per glomerulus and thus average responses rather than single neuron responses are observed. Also, deliberate staining of LNs alone is difficult and thus no distinct conclusions on the dynamic involvement of LNs in the AL computations could be drawn. Finally, the imaging techniques used to measure glomerular activity could only resolve signals from the superficially located T1 glomeruli but not from T2-4 glomeruli. Thus, the odor response activity of local populations of $\mathrm{m}$-PNs could not be tested in any of the mentioned studies. To overcome these limitations we carried out intracellular recordings. This allowed to monitor the activity of single identified neuron types (1-PNs, m-PNs, LNs) with single AP resolution and permitted an analysis of the intracellular membrane potential. We preferentially targeted axons in the median antennocerebralis tract to specifically study $\mathrm{m}-\mathrm{PN}$ function. Taken together, the combined results from $\mathrm{Ca}$-imaging and single neuron electrophysiology contribute to a refined picture of odorinduced processing in the honeybee AL.

\section{RAPID DUAL ENCODING OF ODOR IDENTITY IN THE PN ENSEMBLE}

Our analyses indicate that the PN population in the honeybee encodes odor identity in the firing pattern across activated and inactivated $\mathrm{PNs}$ as well as in the spatial pattern of relative response latencies in the activated PN ensemble. The spatial pattern of PN firing rates evolved rapidly after stimulus onset. Different odors result in a significantly different pattern within few tens of milliseconds as demonstrated by our time-resolved measure of vector distance $d$. Ninety percent of the maximum distance was reached after about $150 \mathrm{~ms}$. This result is in line with previous studies in the locust where odor classification in extracellularly recorded PN ensembles reached the near-maximum after 100-150 ms (Mazor and Laurent, 2005), in Drosophila where odor tuning in individual PNs was strongest in the period of 130-230 ms after stimulus onset (Wilson et al., 2004), and in Bombyx mori where a maximal separation of the PN population activity was reached 100-150 ms after response onset (Namiki and Kanzaki, 2008). Note, that we used a non-causal kernel function for firing rate estimation (see Section "Materials and Methods"). Strict causality for $d$ in Figures 4 and 5 ( $\tau=25 \mathrm{~ms}$ ) adds $42 \mathrm{~ms}$ to the estimated times. Both aspects of this coding scheme, rate and latency, also satisfy the behavioral observation of fast odor discrimination within $<200 \mathrm{~ms}$ (Pamir et al., 2008; Wright et al., 2009).

Stimulus encoding by the temporal pattern of stimulus-response latencies has been postulated for different systems (e.g., Chase and Young, 2007; Hopfield, 1995). In the olfactory system of rodents, it was recently shown that the response latencies of mitral cells in the olfactory bulb are odor-specific (for review see Schaefer and Margrie, 2007). The benefits of a latency code are obvious. By definition, the response onset latencies represent the first datum of an ensemble response. A subset of early responding neurons will yield an initially incomplete representation of odor identity that will be available much sooner than the average onset latency, which we estimated to be $\sim 130 \mathrm{~ms}$ (cf. Figures 2 and 6; note that our method has a tendency of slightly overestimating absolute latencies, see Section "Materials and Methods"). Moreover, the pattern of response latencies might readily yield a concentration-invariant representation of odor identity. At the same time, it seems difficult to implement a reliable latency code for both, stimulus identity and concentration. We may thus hypothesize that the firing rate pattern across the complete PN population acts complementary to the latency code and, with a short delay, provides a refined code of odor identity and concentration. 
From a functional point of view the important question is whether the information present in the ensemble latency pattern is actually used in the next stage of the insect olfactory system, the MB and the LH, and how a useful read out could be accomplished. For rodents it was hypothesized that downstream neurons could read out a latency code through specific delays that involve multi-synaptic transmission and subsequent coincidence detection. However, there are two reasons why the mechanisms of transmitting and reading a latency code are likely to be fundamentally different in insects. In rodents, dynamics of olfactory coding and perception are naturally determined by the sniff cycle, which itself can be controlled by the animal during active sensing (Schaefer and Margrie, 2007; Wesson et al., 2008). In insects, such a dominant rhythm does not exist. Moreover, the anatomical connection scheme is much more complex in rodents than in insects.

In the honeybee, PNs provide input to yet unidentified neurons in the $\mathrm{LH}$ and to the Kenyon cells (KCs), the principal cells of the MB. Here the relatively small number of PNs ( 950 in the honeybee) diverge onto a much larger subpopulation of the total $\sim 160,000 \mathrm{KCs}$ that are assumed to integrate olfactory information. Conversely, each of these KCs receives convergent input from a number of PNs. For the so-called clawed KCs this number was estimated in the order of 10 (Szyszka et al., 2005). Recently (Turner et al., 2008) provided a similar estimate of about 10 15 PN inputs per $\mathrm{KC}$ for the Drosophila. In locust the statistics of convergence might be different (Turner et al., 2008). There, each KC receives input from $\sim 50 \%$ of the 800 PNs with small individual synaptic weights (Jortner et al., 2007). Based on results in the locust we may assume that only a small proportion of KCs respond to a given odor (spatial sparseness, Perez-Orive et al., 2002). Individual KCs responses to single odor stimuli are brief with only a few spikes (temporal sparseness, Perez-Orive et al., 2002; Szyszka et al., 2005). In the locust a mechanism of feedforward inhibition was suggested to explain truncation of $\mathrm{KC}$ responses (Assisi et al., 2007; Perez-Orive et al., 2002). In the bee this may be a result of the inhibitory feedback onto the presynaptic boutons of KCs as revealed by EM studies (Ganeshina and Menzel, 2001) and $\mathrm{Ca}^{2+}$ imaging of the PN boutons (N. Yamagada, personal communication). If we assume a truncated integration time window for KCs, then a KC may detect the first near-coincident inputs from synchronously activated PNs, while desynchronized or delayed inputs may fail to elicit a response. Thus, KC responses might strongly emphasize dynamic changes of the olfactory input, i.e., changes in odorant composition and concentration. In our experimental setting where we probed the responses to a single static stimulus, this property would readily translate the response latency pattern at the level of PNs into a spatio-temporal pattern of briefly activated KCs in response to the stimulus onset, while the static presence of that stimulus would be largely ignored. In a natural environment, olfactory input changes dynamically (Budick and Dickinson, 2006; Vickers, 2000; Vickers et al., 2001). This would lead to a dynamic pattern of PN activation and inactivation and thus to a dynamically changing composition of the activated KC population that follows significant transients in the input. This scenario predicts that under dynamic input conditions, the number of activated $\mathrm{KC}$ may remain low at any given point in time, i.e., preserve a sparse spatial representation in the high-dimensional space of KCs. The spiking activity of individual
KCs, however, would increase and exhibit a more complex temporal pattern as was observed for temporally overlapping odor presentations in the locust (Broome et al., 2006).

\section{LOCAL COMPUTATION IN THE AL NETWORK}

As to date, our anatomical and functional picture of the AL network with its estimated $4000 \mathrm{LNs}$ is still incomplete. Other than in Drosophila (Wilson and Laurent, 2005) but similar to lobsters and moths (Christensen et al., 1993; Schmidt and Ache, 1996) the circuitry of the honeybee AL involves two morphological distinct types of spiking inhibitory LNs that are distinct by their heterogeneous and homogenous branching patterns (Fonta et al., 1993). Moreover, there exists a division into a GABAergic neuron population (Bicker et al., 1993; Sachse and Galizia, 2002; Sachse et al., 2006) and neurons that may involve histaminergic or/and glutaminergic transmission (Barbara et al., 2005; Bicker et al., 1988; Sachse et al., 2006). The existence of excitatory neurons has recently been reported in Drosophila (Olsen et al., 2007; Root et al., 2007; Shang et al., 2007). Their existence has also been predicted for the honeybee AL for various reasons (Malaka et al., 1995), but clear experimental evidence is still lacking. Several of our observations support this hypothesis. First, we report that the $\mathrm{PN}$ response onset, on average, is delayed by about $60 \mathrm{~ms}$ with respect to the faster interneuron response onset (cf. Figure 6D). This fits well to the assumption that PN activation is at least to some extent mediated via excitatory interneurons. Second, excitatory input from LNs can explain how fast responding inhibitory interneurons could effectively suppress responses in PNs before excitatory input drives PN firing (cf. Figure 6A). Third, we observed broad response profiles of individual uniglomerular l-PN and $\mathrm{m}-$ PNs across chemically diverse compounds, an observation that has been made repeatedly in insects. This broad tuning could readily be explained if excitation is mediated by LNs (Olsen et al., 2007). Note that the existence of excitatory interneurons would imply that the individual identified LNs analyzed in our study could be of either type, inhibitory or excitatory.

The most apparent effect of local computation in the AL is the mixture suppression effect where individual uniglomerular 1-PNs are inhibited in response to a mixture but not to its individual components. This is supported by a recent $\mathrm{Ca}^{2+}$ imaging study of $\mathrm{PN}$ boutons in the honeybee that suggests that odor complexity is decoded by a subset of KCs preferentially postsynaptic to l-PN boutons (N. Yamagada, personal communication). Our results showed that the onset of intracellular inhibition in 1-PNs matched the excitatory response onset of LNs, which in turn was significantly shorter than in PNs (Figure 6). From this we conclude that lateral inhibition is responsible for the observed mixture suppression effect. From $\mathrm{Ca}^{2+}$-imaging studies of T1 glomeruli we know that increasing the number of components in the mixture enhanced suppressive interglomerular interaction (Joerges et al., 1997). What could be the behavioral relevance of a mixture-specific code? For social insects like the honeybee a unique cue on the complexity of an odor might indeed be advantageous. In particular, this could allow to distinguish simple odors that are relevant in inter-individual communication from natural plant odorants of high complexity.

Taken together, our recordings indicate that interglomerular connectivity within the AL is not merely a means to regulate and normalize the overall activation level of PNs. Rather, LNs are 
involved in rapidly processing the OSN input. Thus, our findings complement recent interpretations that have strongly emphasized the importance of the AL network in altering and shaping the PN output (Abbott and Luo, 2007; Bhandawat et al., 2007; Olsen et al., 2007; Schlief and Wilson, 2007; Wilson et al., 2004).

\section{SUPPLEMENTARY MATERIAL}

The Supplemental Data for this article can be found online at www.frontiersin.org/computationalneuroscience/paper/10.3389/ neuro.10/009.2008/

\section{REFERENCES}

Abbott, L., and Luo, S. (2007). A step toward optimal coding in olfaction. Nat. Neurosci. 10, 1342-1343.

Abel, R., and Rybak, J., and Menzel, R. (2001). Structure and response patterns of olfactory interneurons in the honeybee, Apis mellifera. J. Comp. Neurol. 437, 363-383.

Abraham, N. M., Spors, H., Carleton, A., Margrie, T.W., Kuner, T., and Schaefer, A.T. (2004). Maintaining accuracy at the expense of speed: stimulus similarity defines odor discrimination time in mice. Neuron 44 , 865-876.

Arnold, G., Masson, C., and Budharugsa, S. (1985). Comparative study of the antennal lobes and their afferent pathway in the worker bee and the drone Apis mellifera. Cell Tissue Res. 242, 593-605.

Assisi, C., Stopfer, M., Laurent, G., and Bazhenov, M. (2007). Adaptive regulation of sparseness by feedforward inhibition. Nat. Neurosci. 10, 1176-1184.

Balderrama, N., Nunez, J., Guerrieri, F., and Giurfa, M. (2002). Different functions of two alarm substances in the honeybee. J. Comp. Physiol. A 188, 485-491.

Barbara, G., Zube, C., Rybak, J., Gauthier, M., and Grünewald, B. (2005). Acetylcholine, GABA and glutamate induce ionic currents in cultured antennal lobe neurons of the honeybee, Apis mellifera. J. Comp. Physiol. A 191, 823-836.

Bhandawat, V., Olsen, S., Gouwens, N., Schlief, M., and Wilson, R. (2007). Sensory processing in the Drosophila antennal lobe increases reliability and separability of ensemble odor representations. Nat. Neurosci. 10, 1474-1482.

Bicker, G., Kreissl, S., and Hofbauer, H. (1993). Monoclonal antibody labels olfactory and visual pathways in Drosophila and Apis brains. J. Comp. Neurol. 335, 413-424.

Bicker, G., Schaefer, S., Ottersen, O., and Storm-Mathisen, J. (1988). Glutamate-like immunoreactivity in identified neuronal populations of insect nervous systems. J. Neurosci. 8, 2108-2122.

Broome, B., Jayaraman, V., and Laurent, G. (2006). Encoding and decoding of overlapping odor sequences. Neuron $51,467-482$.

Budick, S., and Dickinson, M. (2006). Free-flight responses of Drosophila melanogaster to attractive odors. J. Exp. Biol. 209, 3001-3017.

Carlsson, M., Chong, K., Daniels, W., Hansson, B., and Pearce, T. (2007). Component information is preserved in glomerular responses to binary odor mixtures in the moth Spodoptera littoralis. Chem. Senses 32, 433-443.

Chandra, S., and Smith, B. (1998). An analysis of synthetic processing of odor mixtures in the honeybee (Apis mellifera). J. Exp. Biol. 201, 3113-3121.

Chase, S., and Young, E. (2007). First-spike latency information in single neurons increases when referenced to population onset. Proc. Natl. Acad. Sci. USA 104, 5175-5180. and Hildebrand, J. (1993). Local interneurons and information processing in the olfactory glomeruli of the moth Manduca sexta. J. Comp. Neurophysiol. A 4, 385-399.

Davison, I., and Katz, L. (2007). Sparse and selective odor coding by mitral/tufted neurons in the main olfactory bulb. $J$. Neurosci. 27, 2091-2101.

Deisig, N., Giurfa, M., Lachnit, H., and Sandoz, J. (2006). Neural representation of olfactory mixtures in the honeybee antennal lobe. Eur. J. Neurosci. 24, 1161-1174.

Deisig, N., Lachnit, H., Sandoz, J., Lober, K., and Giurfa, M. (2003). A modified version of the unique cue theory accounts for olfactory compound processing in honeybees. Learn. Mem. 10, 199-208.

Duchamp-Viret, P., Duchamp, A., and sensory neurons simultaneously integrate the components of an odour mixture. Eur. J. Neurosci. 10, 2690-2696.

Esslen, J., and Kaissling, K. (1976). Zahl und Verteilung antennaler Sensillen
Christensen, T., Waldrop, B., Harrow, I., Chaput, M. (2003). Single olfactory

\section{ACKNOWLEDGEMENTS}

We thank Jürgen Rybak for his helpful comments on an earlier version of this manuscript. We are grateful to Martin Strube, Paul Szyszka, Silke Sachse and Tilman Franke for valuable discussion. We thank Stefan Rotter for his advice in designing our data analysis. Sabine Krofczik was funded by the Deutsche Forschungsgemeinschaft through grant Me 365/27-1 to Randolf Menzel. Additional funding was received from the German Federal Ministry of Education and Research (grant 01GQ0413 to BCCN Berlin).

bei der Honigbiene Apis mellifera $\mathrm{L}$. Zoomorphologie 83, 227-251.

Flanagan, D., and Mercer, A. (1989). An atlas and 3-D reconstruction of the antennal lobes in worker honey bee, Apis mellifera L. (Hymenoptera: Apidae). Int. J. Insect Morphol. Embryol. 18, 145-159.

Fonta, C., Sun, X., and Masson, C. (1993). Morphology and spatial distribution of bee antennal lobe interneurones responsive to odours. Chem. Senses $18,101-119$.

Galizia, C., and Kimmerle, B. (2004) Physiological and morphological characterization of honeybee olfactory neurons combining electrophysiology, calcium imaging and confocal microscopy. J. Comp. Physiol. A 190, 21-38.

Galizia, C., McIlwrath, S., and Menzel, R. (1999). A digital three-dimensional atlas of the honeybee antennal lobe based on optical sections acquired by confocal microscopy. Cell Tissue Res. 295, 383-394.

Galizia, C., and Menzel, R. (2000). Odour perception in honeybees: coding information in glomerular patterns. Curr. Opin. Neurobiol. 10, 504-510.

Ganeshina, O., and Menzel, R. (2001) GABA-immunoreactive neurons in the mushroom bodies of the honeybee: an electron microscopic study J. Comp. Neurol. 437, 335-349.

Giraudet, P., Berthommier, F., and Chaput, M. (2002). Mitral cell temporal response patterns evoked by odor mixtures in the rat olfactory bulb. J. Neurophysiol. 88, 829-838.

Guerrieri, F., Schubert, M., Sandoz, J., and Giurfa, M. (2005). Perceptual and neural olfactory similarity in honeybees. PLoS Biol. 3, e60.

Heil, J. E., Oland, L. A., and Lohr, C. (2007). Acetylcholine-mediated axonglia signaling in the developing insect olfactory system. Eur. J. Neurosci. 26, 1227-1241.

Hopfield, J. (1995). Pattern recognition computation using action potential timing for stimulus representation. Nature 376, 33-36.

Joerges, J., Kuettner, A., Galizia, C., and Menzel, R. (1997). Representation of odours and odour mixtures visualized in the honeybee brain. Nature 387, 61-69.

Jortner, R., Farivar, S., and Laurent, G. (2007). A simple connectivity scheme for sparse coding in an olfactory system. J. Neurosci. 27, 1659-1669.

Kelber,C., Rössler,W., and Kleineidam, C. J. (2006). Multiple olfactory receptor neurons and their axonal projections in the antennal lobe of the honeybee Apis mellifera. J. Comp. Neurol. 496, 395-405.

Kirschner, S., Kleineidam, C., Zube, C., Rybak, J., Grünewald, B., and Roessler, W. (2006). Dual olfactory pathway in the honeybee, Apis mellifera. J. Comp. Neurol. 499, 933-952.

Lei, H., Mooney, R., and Katz, L. (2006). Synaptic integration of olfactory information in mouse anterior olfactory nucleus. J. Neurosci. 26, 12023-12032.

Lin, D., Shea, S., and Katz, L. (2006) Representation of natural stimuli in the rodent main olfactory bulb. Neuron 50, 937-949.

Livermore, A., and Laing, D. (1998). The influence of odor type on the discrimination and identification of odorants in multicomponent odor mixtures. Physiol. Behav. 65, 311-320.

Malaka, R., Ragg, T., and Hammer, M. (1995). Kinetic models of odor transduction implemented as artificial neural networks. Simulations of complex response properties of honeybee olfactory neurons. Biol. Cybern. 73, 195-207.

Mazor, O., and Laurent, G. (2005) Transient dynamics versus fixed points in odor representations by locust antennal lobe projection neurons. Neuron 48, 661-673.

Meier, R., Egert, U., Aertsen, A., and Nawrot, M. (2008). FIND - a unified framework for neural data analysis. Neural Netw. 21, 1085-1093.

Mobbs, P. (1982). The brain of the honeybee Apis mellifera I. The connections and spatial organization of the mushroom bodies. Philos. Trans. R. Soc. Lond., B, Biol. Sci. 298, 309-354. 
Müller, D., Abel, R., Brandt, R., Zoeckler, M., and Menzel, R. (2002). Differential parallel processing of olfactory information in the honeybee, Apis mellifera L. J. Comp. Physiol. A 188, 359-370.

Namiki, S., and Kanzaki, R. (2008). Reconstructing the population activity of olfactory output neurons that innervate identifiable processing units. Front. Neural Circuits 2, 1 [Epub ahead of print].

Nawrot, M., Aertsen, A., and Rotter, S. (1999). Single-trial estimation of neuronal firing rates: from single-neuron spike trains to population activity. J. Neurosci. Methods 94, 81-92.

Nawrot, M., Aertsen, A., and Rotter, S. (2003). Elimination of response latency variability in neuronal spike trains. Biol. Cybern. 88, 321-334.

Olsen, S. R., Bhandawat, V., and Wilson, R. I. (2007). Excitatory interactions between olfactory processing channels in the Drosophila antennal lobe. Neuron 54, 89-103.

Pamir, E., Schmuker, M., Strube-Bloss, M., Menzel, R., and Nawrot, M. P. (2008). Detection of odor-specific response latencies in the honeybee olfactory system. Front. Comput. Neurosci. Bernstein Symposium 2008. doi: 10.3389/conf.neuro.10.2008.01.103.

Parzen,E. (1962). On estimation of a probability density function and mode. Ann. Math. Stat. 33, 1065-1076.

Perez-Orive, J., Mazor, O., Turner, G., Cassenaer, S., Wilson, R., and Laurent, G. (2002). Oscillations and sparsening of odor representations in the mushroom body. Science 297, 359-365.

Pichersky, E., and Gershenzon, J. (2002). The formation and function of plant volatiles: perfumes for pollinator attraction and defense. Curr. Opin. Plant Biol. 5, 337-343.
Root, C. M., Semmelhack, J. L., Wong, A. M., Flores, J., and Wang, J. W. (2007). Propagation of olfactory information in Drosophila. Proc. Natl. Acad. Sci. USA 104, 11826-11831.

Rybak, J., and Eichmueller, S. (1993). Structural plasticity of an immunocytochemically identified set of honeybee olfactory interneurones. Acta Biol. 1, 61-65.

Sachse, S., and Galizia, C. (2002). Role of inhibition for temporal and spatial odor representation in the olfactory output neurons: a calcium imaging study. J. Neurophysiol. 87, 1106-1117.

Sachse, S., Peele, P., Silbering, A., Guehmann, M., and Galizia, C. (2006). Role of histamine as a putative inhibitory transmitter in the honeybee antennal lobe. Front. Zool. 3, 22.

Sachse, S., Rappter, A., and Galizia, C. (1999). The spatial representation of chemical structures in the antennal lobe of honeybees: steps towards the olfactory code. Eur. J. Neurosci. 11, 3970-3982.

Savitzky, A., and Golay, M. (1964). Smoothing and differentiation of data by simplified least squares procedures. Anal. Chem. 36, 1627-1639.

Schaefer, A., and Margrie, T. (2007). Spatiotemporal representations in the olfactory system. Trends Neurosci. 30, 92-100.

Schlief, M., and Wilson, R. (2007). Olfactory processing and behavior downstream from highly selective receptor neurons. Nat. Neurosci. 10, 623-630.

Schmidt, M., and Ache, B. (1996). Processing of antennular input in the brain of the spiny lobster, Panulirus argus. II. The olfactory pathway. J. Comp. Physiol. A 178, 605-628.

Shang, Y., Claridge-Chang, A., Sjulson, L., Pypaert, M., and Miesenboeck, G.
(2007). Excitatory local circuits and their implications for olfactory processing in the fly antennal lobe. Cell 128, 601-612.

Silbering, A., and Galizia, C. (2007). Processing of odor mixtures in the Drosophila antennal lobe reveals both global inhibition and glomerulusspecific interactions. J. Neurosci. 27, 11966-11977.

Strube-Bloss, M., Nawrot, M. P., and Menzel,R. (2008). Odor Learning Leads to the Recruitment of Previously NonResponding Alpha-Lobe Extrinsic Neurons in the Honeybee. Program No. 792.17.2008. Neuroscience Meeting Planner. Washington, DC Society for Neuroscience. Online.

Szyszka, P., Ditzen, M., Galkin, A., Galizia, C., and Menzel, R. (2005). Sparsening and temporal sharpening of olfactory representations in the honeybee mushroom bodies. J. Neurophysiol. 94, 3303-3313.

Tabor, R., Yaksi, E., Weislogel, J., and Friedrich, R. (2004). Processing of odor mixtures in the zebrafish olfactory bulb. J. Neurosci. 24, 6611-6620.

Turner, G., Bazhenov, M., and Laurent, G. (2008). Olfactory representations by Drosophila mushroom body neurons. J. Neurophysiol. 99, 734-746.

Valenticic, T., Kralj, J., Stenovec, M., Koce, A., and Caprio, J. (2000). The behavioral detection of binary mixtures of amino acids and their individual components by catfish. J. Exp. Biol. 203, 3307-3317.

Vickers, N. (2000). Mechanisms of animal navigation in odor plumes. Biol. Bull. 198, 203-212.

Vickers, N., Christensen, T., Baker, T. and Hildebrand, J. (2001). Odourplume dynamics influence the brain's olfactory code. Nature 410 , 466-470.
Wesson, D., Carey, R., Verhagen, J., and Wachowiak, M. (2008). Rapid encoding and perception of novel odors in the rat. PLoS Biol. 6, e82.

Wilson, R., and Laurent, G. (2005). Role of GABAergic inhibition in shaping odor-evoked spatiotemporal patterns in the Drosophila antennal lobe. J. Neurosci. 25, 9069-9079.

Wilson, R., Turner, G., and Laurent, G. (2004). Transformation of olfactory representations in the Drosophila antennal lobe. Science 303, 366-370.

Wright, G.A.,Carlton,M., and Smith, B. H. (2009). A honeybee's ability to learn, recognize, and discriminate odors depends upon odor sampling time and concentration. Behav. Neurosci. $123,36-43$.

Zou,Z., and Buck,L. (2006).Combinatorial effects of odorant mixes in the olfactory cortex. Science 311, 1477-1481.

Conflict of Interest Statement: The authors declare that the research was conducted in the absence of any commercial or financial relationships that could be construed as a potential conflict of interest.

Received: 02 June 2008; paper pending published: 24 July 2008; accepted: 17 December 2008; published online: 15 January 2009.

Citation: Krofczik S, Menzel Rand Nawrot MP (2008) Rapid odor processing in the honeybee antennal lobe network. Front. Comput. Neurosci. (2009) 2:9. doi: 10.3389/neuro.10.009.2008

Copyright (๑) 2009 Krofczik, Menzel and Nawrot. This is an open-access article subject to an exclusive license agreement between the authors and the Frontiers Research Foundation, which permits unrestricted use, distribution and reproduction in any medium, provided the original authors and source are credited. 